\title{
Radiographic analysis of the effects of first metatarsal rotation in hallux valgus surgery
}

\author{
Alexandre de Andrade Budin ${ }^{(\mathbb{D})}$, Helencar Ignacio ${ }^{(\mathbb{D})}$, Marcio Gomes Figueiredo ${ }^{(\mathbb{D})}$ \\ 1. Faculdade de Medicina de São José do Rio Preto, São Paulo, SP, Brazil.
}

\begin{abstract}
Objective: To evaluate whether the initial degree of metatarsal rotation interferes with the surgical correction of severe hallux valgus. Methods: A retrospective study was performed using weight-bearing AP radiographs to measure first metatarsal rotation based on the shape of the lateral edge of the metatarsal head and the hallux valgus (HVA) and intermetatarsal (IMA) angles. Participants were then classified into two groups. Those with less rotational deformity were placed in the negative pronation group, while those with greater rotational deformity were placed in the positive pronation group. Mean HVA and IMA correction were calculated and compared between groups. Participants underwent the modified Lapidus procedure with correction of pronation.

Results: Data were collected for 26 feet with hallux valgus. The negative and positive pronation groups contained 14 and 12 feet, respectively. Successful surgical correction of pronation was observed in 11 of the $12 \mathrm{feet}$, which were ultimately classified in the negative pronation group based on postoperative radiographs. The negative pronation group showed a mean difference of $15.05^{\circ}$ in the $\mathrm{HVA}$ and $4.20^{\circ}$ in the IMA. The positive pronation group showed a mean difference of $14.22^{\circ}$ in the HVA and $3.2^{\circ}$ in the IMA. These values did not significantly differ between groups.
\end{abstract}

Conclusion: The initial degree of pronation does not affect the degree of angular correction as long as metatarsal rotation is also addressed.

\section{Level of Evidence IV; Diagnostic Studies; Case Series.}

Keywords: Hallux valgus/diagnostic imaging; Hallux valgus/surgery; Metatarsal bones/diagnostic imaging; Rotation; Pronation; Range of motion, articular.

\section{Introduction}

Hallux valgus is a common condition of the foot consisting of a multiplanar deformity that occurs most frequently in women starting in the fourth decade of life. Up to $90 \%$ of patients with hallux valgus show deformities in the coronal plane, with metatarsal rotation and, consequently, pronation of the hallux ${ }^{(1)}$. This deformity plays an important role in disease progression, as it is frequently accompanied by sesamoid subluxation and an imbalance in muscle forces in the first phalangeal-metatarsal joint ${ }^{(2)}$. If uncorrected, metatarsal rotation can increase the likelihood of recurrence after surgery, as demonstrated by studies of hallux pronation and its impact on disease recurrence after surgical treatment ${ }^{(3-5)}$.
The shape of the lateral cortical surface of the metatarsal head has been identified as an important factor to be considered in pre- and postoperative anteroposterior (AP) radiographs of the foot in orthostasis since a rounded metatarsal head is associated with higher rates of hallux valgus recurrence, as identified by Okuda et al. ${ }^{(6)}$. The author also noted that alterations in the lateral cortical surface of the metatarsal head could be related to the degree of metatarsal pronation, with rounder surfaces indicating a higher degree of pronation ${ }^{(6)}$. The method described by Okuda et al. ${ }^{(7)}$ to classify the shape of the lateral cortical surface was adopted in subsequent studies, such as that of Yamaguchi et al. ${ }^{(8)}$, who found this variable to be associated with metatarsal rotation, confirming the correlation between these factors using digitally reconstructed radiographs from axial computed tomography (CT) scans.
Study performed at the Faculdade de Medicina de São José do Rio Preto, São Paulo, Brazil.

Correspondence: Alexandre de Andrade Budin. 5544 Brigadeiro Faria Lima Av., Vila Sao Jose, Sao Jose do Rio Preto, SP, Brazil, Zip Code: 15090-000. E-mail: alexandrebudin23@gmail.com. Conflicts of interest: none. Source of funding: none. Date received: March 15, 2021. Date accepted: April 09, 2021. Online: April 30, 2021

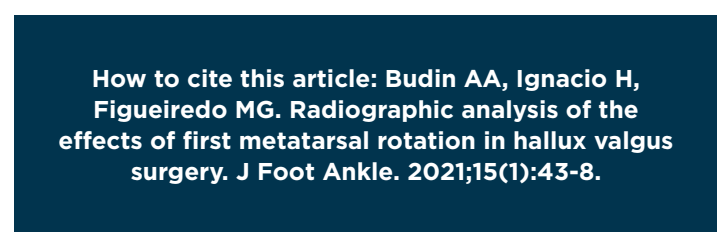


The surgical correction of these rotational deformities has been described in previous studies ${ }^{(9-11)}$. Dayton et al. described improvement in the angular measurements of hallux valgus with the modified Lapidus procedure ${ }^{(12)}$ where the original technique was altered to focus specifically on the correction of metatarsal rotation ${ }^{(11)}$.

The aim of this study was to evaluate whether the degree of pronation observed in preoperative radiographs of severe hallux valgus interferes with the corrective effects of the modified Lapidus procedure on the hallux valgus (HVA) and intermetatarsal (IMA) angles ${ }^{(12)}$.

\section{Methods}

\section{Patients}

A study was conducted based on the weight-bearing AP radiographs of the foot of patients submitted to surgical treatment of hallux valgus using the Lapidus technique. The study was approved by a research ethics committee. All patients had severe hallux valgus with $\mathrm{HVA}>40^{\circ}$ or IMA $>16^{\circ}$. Pre- and postoperative images, obtained two months to one year after surgery, were evaluated. Surgeries were performed by the same foot and ankle surgery team, including two senior foot and ankle surgeons, between 2012 and 2019. After reviewing medical records, the research team identified 26 feet treated using the modified Lapidus technique. The feet belonged to 23 patients, 22 female and 1 male, with a mean age of 60.18 years (SD, \pm 15.58 ).

The technique involves a dorsal approach to the tarsometatarsal joint, with careful dissection to preserve vascularization. An osteotomy was performed at the base of the first metatarsal with the resection of all cartilage to allow for the correction of deformities in the sagittal and transverse planes. Rotational deformities can be corrected by inserting a 2.0 $\mathrm{mm}$ Kirschner wire perpendicular to the first metatarsal to be used as a joystick to control supination of the first metatarsal while correcting the transverse plane. The wire is then positioned and fixed to the lateral surface of the second metatarsal. This metatarsal de-rotation technique was first used by the surgical team in 2016. Prior to this point, the primary goal of the perpendicular placement of the Kirschner wire was not to achieve de-rotation but to increase stability and allow for early ambulation. The radiographic analysis of cases of severe hallux valgus that received surgical treatment before 2016 did consider the possibility of inadvertent de-rotation of metatarsal pronation. Four of the feet included in this study had undergone surgery prior to 2016.

\section{Radiographic analysis}

The radiographic assessment of first metatarsal rotation, which corresponds to the degree of hallux pronation, was performed as described by Okuda et al. ${ }^{(7)}$ with modifications by Yamaguchi et al. ${ }^{(8)}$. Printed radiographic images and a goniometer were used to measure the distance from the center to the lateral edge of the metatarsal head. The goniometer was placed over the center of the metatarsal head, with the concentric circles outlining the metatarsophalangeal joint. The maximum distance of the circle to the lateral edge of the metatarsal head was measured. Cases in which this distance was greater than $2 \mathrm{~mm}$ were classified as group A, or angular. When this distance was between 1 and $2 \mathrm{~mm}$, the case was classified as intermediate, or group B. If the circle perfectly outlined the metatarsal head or the distance was smaller than $1 \mathrm{~mm}$, the case was classified as group $C$, or round.

Patients were divided into groups $A, B$, and $C$ based on the shape of the metatarsal head in preoperative weight-bearing AP radiographs. Group C patients had a rounded metatarsal head, corresponding to the greatest degree of rotation of the first metatarsal. These individuals were classified in a positive pronation group, while patients in groups A and B were combined into a negative pronation category due to their smaller degree of hallux pronation (Figure 1). The two groups were subsequently compared using statistical methods.

Participants were classified into positive and negative pronation groups based on the degree of pronation observed in preoperative and postoperative images. The degree of surgical correction was calculated based on the HVA and IMA values determined using preoperative and postoperative weight-bearing AP radiographs. The mean values of preoperative and postoperative measurements were then calculated for each group. The correlation between the degree of correction and the degree of metatarsal rotation was also examined.
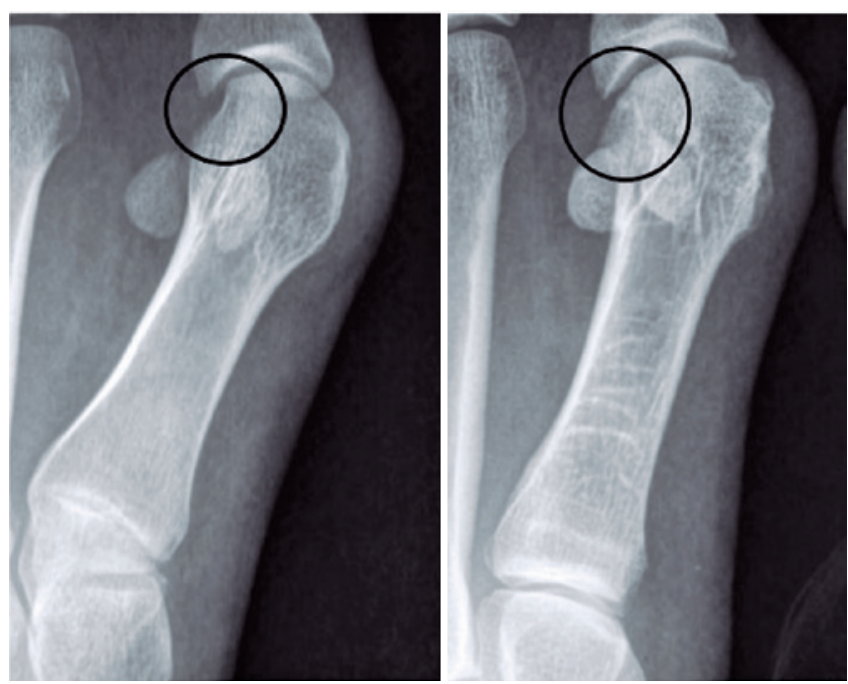

Figure 1. On the left, the lateral edge has an angular shape, indicating less pronation (negative pronation). On the right, the lateral edge is rounded, indicating greater pronation (positive pronation). 


\section{Statistical analysis}

Preoperative and postoperative HVA and IMA values were calculated for all patients. The difference between pre- and postoperative values was then determined, and mean values were compared between the negative $(A+B)$ and positive $(C)$ pronation groups using statistical methods.

Data were analyzed using the Statistical Package for the Social Sciences (SPSS, IBM, version 24.0) and GraphPad Instat 3.10 (2009).

Categorical variables were summarized as frequencies and percentages. Quantitative variables were analyzed using measures of central tendency and dispersion. Normality was tested using the Kolmogorov-Smirnov test. Inferential analysis was conducted using Student's T-tests and Chi-square tests. Associations between quantitative variables were examined using Pearson correlation coefficients. The coefficients ( $r$ ) were classified as described by Dancey and Reidy (2005), in the following categories:

$$
\begin{aligned}
& r=0.10 \text { to } 0.30 \text { (weak) } \\
& r=0.40 \text { to } 0.60 \text { (moderate) } \\
& r=0.70 \text { to } 1 \text { (strong) }
\end{aligned}
$$

In all analyses, results were considered significant at $\mathrm{p} \leq 0.05$.

\section{Results}

Measurements of metatarsal rotation revealed that 12 feet with hallux valgus had a high degree of pronation. These were placed in the positive pronation group (group C), while the remaining 14 feet were placed in the negative pronation group (groups $A+B$ ). Eleven of the 12 feet originally classified as group $C$ were reclassified as group $B$ after the analysis of postoperative radiographs, which demonstrated a less rounded metatarsal head (Figure 2). One foot in group $C$ did not
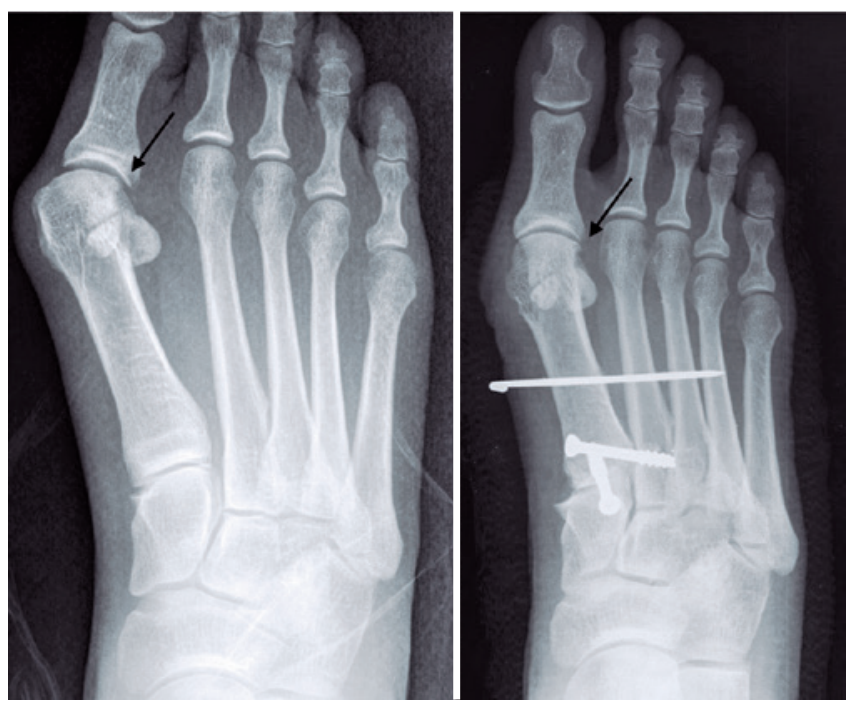

Figure 2. Rotational correction, as shown in pre- and postoperative radiographs, demonstrated by the less rounded appearance of the metatarsal head. show radiographic improvement of the metatarsal head shape after surgery and retained its classification. One patient in group B was reclassified to group A. All patients in group A maintained their classification. The analysis of postoperative radiographs of the 4 feet treated prior to 2016 resulted in 3 feet being classified into the negative pronation group and 1 in the positive pronation group. After surgery, however, the latter was reclassified into the negative pronation group.

Preoperative measurements for the sample as a whole revealed a mean HVA of $36.58^{\circ}\left(\mathrm{SD}, \pm 9.22^{\circ}\right.$ ) and a mean IMA of $14.98^{\circ}\left(\mathrm{SD}, \pm 3.40^{\circ}\right.$ ). On postoperative measurements, the mean HVA was $21.91^{\circ}\left(\mathrm{SD}, \pm 10.20^{\circ}\right.$ ) and the mean IMA was $11^{\circ}\left(\mathrm{SD}, \pm 3.25^{\circ}\right)$. Preoperative measurements were then calculated for each group of participants. Those in the pronation-positive group had a mean HVA of $39.99^{\circ}$ (SD, $\pm 8.93^{\circ}$ ) and an IMA of $15.62^{\circ}\left(\mathrm{SD}, \pm 3.43^{\circ}\right)$ while those in the pronation-negative group had a mean HVA of $34.28^{\circ}$ (SD, $\pm 8.48^{\circ}$ ) and an IMA of $14.42^{\circ}\left(S D, \pm 3.40^{\circ}\right)$. Preoperative HVA and IMA values did not significantly differ between groups $(p=0.058$ and $p=0.1915$, respectively).

In the pronation-negative group $(A+B)$, the mean difference between pre- and postoperative HVA was $15.05^{\circ}$ while that of IMA was $4.20^{\circ}$. The pronation-positive group (C) showed a mean difference of $14.22^{\circ}$ in the HVA and $3.2^{\circ}$ in the IMA. A Student's T-test of the differences in pre- and postoperative HVA between pronation negative $(A+B)$ and positive (C) groups yielded $p=0.7343$, indicating that these values did not significantly differ from one another. The results of between-group comparisons of changes in IMA values was similarly nonsignificant, with $p=0.8419$. The results of the statistical analysis are shown in table 1.

Table 1. Statistical analysis of the results obtained from the cal-

\begin{tabular}{|c|c|c|c|c|}
\hline Col. title & $\begin{array}{l}\text { Negative } \\
\text { pronation } \\
\text { group (IMA) }\end{array}$ & $\begin{array}{c}\text { Negative } \\
\text { pronation } \\
\text { group (HVA) }\end{array}$ & $\begin{array}{c}\text { Positive } \\
\text { pronation } \\
\text { group (IMA) }\end{array}$ & $\begin{array}{c}\text { Positive } \\
\text { pronation } \\
\text { group (HVA) }\end{array}$ \\
\hline Mean & -4.207142857 & 15.05 & -3.716666667 & 14.225 \\
\hline $\begin{array}{l}\text { Standard } \\
\text { deviation } \\
\text { (SD) }\end{array}$ & 2.542 & 8.271 & 4.596 & 12.461 \\
\hline $\begin{array}{l}\text { Std. error of } \\
\text { mean (SEM) }\end{array}$ & 0.6794 & 2.211 & 1.327 & 3.597 \\
\hline $\begin{array}{l}\text { 95\% Cl lower } \\
\text { limit }\end{array}$ & -5.675 & 10.275 & -6.637 & 6.308 \\
\hline $\begin{array}{l}95 \% \mathrm{Cl} \text { upper } \\
\text { limit }\end{array}$ & -2.74 & 19.825 & -0.7965 & 22.142 \\
\hline Minimum & -8.5 & 1.2 & -11.4 & 0.1 \\
\hline $\begin{array}{l}\text { Median ( } 50^{\text {th }} \\
\text { percentile) }\end{array}$ & -3.65 & 14.8 & -2.8 & 11.75 \\
\hline Maximum & -0.5 & 27.8 & 3.4 & 40.5 \\
\hline $\begin{array}{l}\text { Normality } \\
\text { test KS }\end{array}$ & 0.135 & 0.1235 & 0.1696 & 0.1654 \\
\hline $\begin{array}{l}\text { Normality } \\
\text { test } p \text { value }\end{array}$ & $>0.10$ & $>0.10$ & $>0.10$ & $>0.10$ \\
\hline $\begin{array}{l}\text { Passed } \\
\text { normality } \\
\text { test? }\end{array}$ & Yes & Yes & Yes & Yes \\
\hline
\end{tabular}
culation of angle improvement in patients with less pronation (negative pronation) and greater pronation (positive pronation) 
Pearson correlations were used to evaluate the association between pronation-positive and pronation-negative groups, and the difference in pre- and postoperative IMA and VHA values. The pronation-negative group was assigned a value of 1 while the pronation-positive group was given a value of 2 . The correlation between IMA values and the degree of pronation was approximated by an ascending line (Figure 3 ). The correlation between pronation and HVA values, on the other hand, yielded a descending line (Figure 4). The $r$ values for these correlations were $0.06992(p=0.7343)$ for the IMA and $0.04111(p=0.84190)$ for the HVA values.

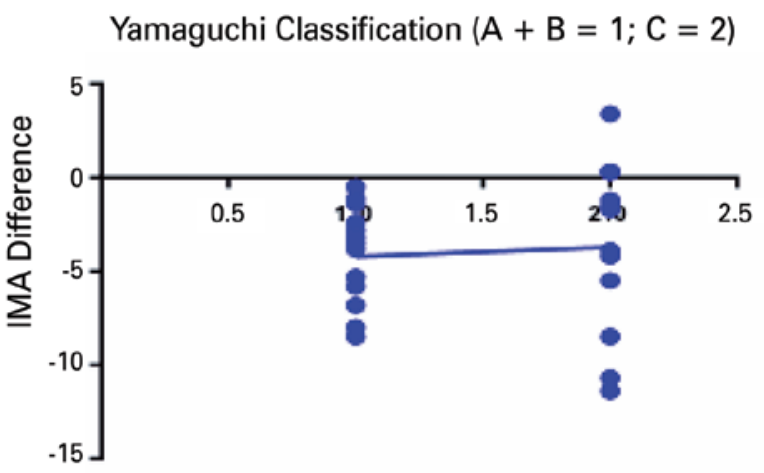

Figure 3. Upward-sloping line demonstrating differences in the IMA. The $A+B$ group corresponds to the negative pronation group. Group $C$ had greater pronation.

Yamaguchi Classification $(A+B=1 ; C=2)$

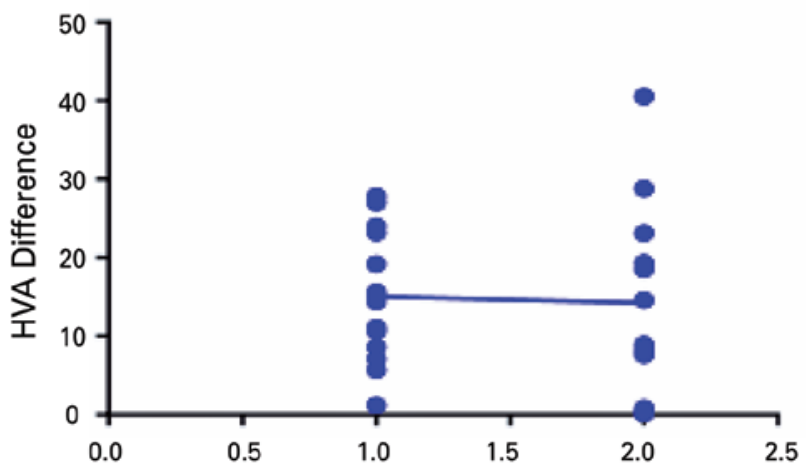

Figure 4. Downward-sloping line demonstrating differences in the HVA. The A+B group corresponds to the negative pronation group. Group $C$ had greater pronation.

\section{Discussion}

Hallux valgus is an illness with a multifactorial pathogenesis, and the degree of rotation of the first metatarsal has become an increasingly important issue in the surgical treatment of this condition ${ }^{(4,7,13)}$. We agree with other authors that the careful evaluation and surgical treatment planning of hallux valgus must consider its three-dimensional nature ${ }^{(14)}$. The presence of a rounded metatarsal head in preoperative radiographs can indicate that a significant degree of pronation is present and must be corrected during surgery. Residual pronation is a risk factor for hallux valgus recurrence, and the presence of a rounded metatarsal head on postoperative radiographs can indicate that the pronation deformity was not corrected ${ }^{(8,9,15)}$. Incomplete sesamoid reduction with lateral subluxation of the sesamoid complex is also associated with higher rates of recurrence and may be directly linked to the incomplete correction of metatarsal rotation, as shown in previous studies ${ }^{(10,16)}$. The surgical technique used in this study aimed to reduce the sesamoid complex under the metatarsal head simultaneously with metatarsal derotation. Sesamoid reduction was not evaluated in this study, as our focus was on the association between metatarsal derotation and angle correction. Nevertheless, in future studies, we plan on evaluating the correlation between incomplete sesamoid reduction and uncorrected rotation.

In the present investigation, we evaluated the results of 8 years of use of the modified Lapidus technique in the treatment of hallux valgus ${ }^{(12)}$. Starting in 2016, the correction of metatarsal rotation garnered special attention as a means of improving angle correction rates. Dayton et al. ${ }^{(11)}$ demonstrated the potential of first metatarsal rotation as a standalone treatment for hallux valgus, and our findings support this hypothesis, as both groups achieved similar correction rates regardless of preoperative differences, especially in HVA values.

The rotation of the first metatarsus was evaluated based on pre- and postoperative weight-bearing AP radiographs of the foot, a method that is simple and inexpensive, and involves the analysis of the shape of the lateral edge of the first metatarsal head, as described in previous studies, such as those of Okuda et al. and Yamaguchi et al. ${ }^{(7,8)}$ The degree of rotation of the first metatarsal can be evaluated using computed tomography ${ }^{(8)}$ or axial radiographs of the first metatarsal(17,18) as described in the literature.

In this study, the results obtained in the two pronation groups did not significantly differ from one another. In the intraoperative period, one of our main concerns is the correction of the rotational deformity of the metatarsal bone. This was achieved in 11 of the 12 cases of hallux valgus with significant pronation (pronation-positive group) in the present study. Since this approach has only been used since 2016, it can be assumed that any rotational correction observed in cases treated before this date was unintentional. In recent years, special attention has also been paid to sesamoid reduction. While this was not evaluated in the present study, as the analysis focused on rotational correction, the authors intend to evaluate this variable in future studies. According to a study by Lee et al. ${ }^{(16)}$, incomplete 
sesamoid reduction is associated with worse radiographic outcomes and increased recurrence. Zitouna et al. ${ }^{(19)}$ concluded that incomplete sesamoid reduction does not necessarily predict poor clinical outcome.

We believe that the length of follow-up in the present study may have been too short to assess recurrence rates in patients submitted to surgical treatment, and suggest that future studies evaluate these rates in longer follow-up periods. Our main goal in this study was to observe whether the initial degree of metatarsal rotation had a negative impact on the outcome of the modified Lapidus technique. In a review on metatarsal pronation, Wagner and Wagner ${ }^{(20)}$ found that the recurrence rate of hallux valgus ranged from 2 to $50 \%$, describing this as a multifactorial complication citing incomplete sesamoid reduction as a main contributing factor. The study in question also found that in $86 \%$ of cases, tibial sesamoid position depended on the degree of metatarsal rotation, further emphasizing the importance of rotational correction in the prevention of recurrence.

The negative trend between the degree of metatarsal rotation and the correction of the HVA in the present study (Figure 2) may indicate an association between the correction of the HVA and metatarsal de-rotation, since the presence of significant pronation may increase the difficulty of surgical correction of the valgus angle. This must be verified in larger samples, since the degree of correction did not differ between groups in the present study, despite the trend toward lower correction rates in patients with greater pronation. Mortier et al. ${ }^{(17)}$ have also demonstrated a possible correlation between pronation and lower correction rates in hallux valgus. We believe that the similarity in IMA and HVA correction between the two groups in the present study is attributable to the correction of pronation in most patients in the positive pronation group.

The upward line corresponding to the association between pronation and IMA correction (Figure 3) suggests that greater pronation is associated with a larger increase in IMA, and consequently, greater potential for correction of this deformity (21-23).

The short follow-up period and small number of cases are possible limitations of this study, as is the potential bias of unintentional correction in cases treated prior to 2016.

\section{Conclusion}

Patients with severe hallux valgus with different degrees of pronation displayed similar degrees of IMA and HVA correction after modified Lapidus surgery.

Authors' contributions: Each author contributed individually and significantly to the development of this article: AAB *(https://orcid.org/O000-0002-13587950) Wrote the article, data collection, survey of the medical records; HI *(https://orcid.org/O000-0002-1179-4809) Wrote the article, approved the final version MGF *(https://orcid.org/0000-0002-5163-1035) Wrote the article, approved the final version. All authors read and approved the final manuscript. *ORCID (Open Researcher and Contributor ID) (iD).

\section{References}

1. Wagner P, Wagner E. Role of Coronal Plane Malalignment in Hallux Valgus Correction. Foot Ankle Clin. 2020;25(1):69-77.

2. Sadamasu A, Yamaguchi S, Kimura S, Ono Y, Sato Y, Akagi R, Sasho T, Ohtori S. Influence of foot position on the measurement of first metatarsal axial rotation using the first metatarsal axial radiographs. J Orthop Sci. 2020;25(4):664-70.

3. Talbot KD, Saltzman CL. Hallucal rotation: a method of measurement and relationship to bunion deformity. Foot Ankle Int. 1997;18(9):550-6.

4. Wagner P, Wagner E. Is the Rotational Deformity Important in Our Decision-Making Process for Correction of Hallux Valgus Deformity? Foot Ankle Clin. 2018;23(2):205-217.

5. Okuda R, Kinoshita M, Yasuda T, Jotoku T, Kitano N, Shima H. Postoperative incomplete reduction of the sesamoids as a risk factor for recurrence of hallux valgus. J Bone Joint Surg Am. 2009;91(7):1637-45.

6. Okuda R, Yasuda T, Jotoku T, Shima H. Supination stress of the great toe for assessing intraoperative correction of hallux valgus. J Orthop Sci. 2012;17(2):129-35.

7. Okuda R, Kinoshita M, Yasuda T, Jotoku T, Kitano N, Shima H. The shape of the lateral edge of the first metatarsal head as a risk factor for recurrence of hallux valgus. J Bone Joint Surg Am. 2007;89(10):2163-72.
8. Yamaguchi S, Sasho T, Endo J, Yamamoto Y, Akagi R, Sato Y, Takahashi K. Shape of the lateral edge of the first metatarsal head changes depending on the rotation and inclination of the first metatarsal: a study using digitally reconstructed radiographs. J Orthop Sci. 2015;20(5):868-74.

9. Okuda R. Proximal Supination Osteotomy of the First Metatarsal for Hallux Valgus. Foot Ankle Clin. 2018;23(2):257-269.

10. Shurnas PS, Watson TS, Crislip TW. Proximal first metatarsal opening wedge osteotomy with a low profile plate. Foot Ankle Int. 2009;30(9):865-72.

11. Dayton P, Feilmeier M, Kauwe M, Hirschi J. Relationship of frontal plane rotation of first metatarsal to proximal articular set angle and hallux alignment in patients undergoing tarsometatarsal arthrodesis for hallux abducto valgus: a case series and critical review of the literature. J Foot Ankle Surg. 2013;52(3):348-54.

12. Sangeorzan BJ, Hansen ST Jr. Modified Lapidus procedure for hallux valgus. Foot Ankle. 1989;9(6):262-6.

13. Kim JS, Young KW. Sesamoid position in hallux valgus in relation to the coronal rotation of the first metatarsal. Foot Ankle Clin. 2018;23(2):219-30.

14. Winson DMG, Perera A. How I use a three-dimensional approach to correct hallux valgus with a distal metatarsal osteotomy. Foot Ankle Clin. 2018;23(2):231-8. 
15. Sadamasu A, Yamaguchi S, Kimura S, Ono Y, Sato Y, Akagi R, Sasho T, Ohtori S. Influence of foot position on the measurement of first metatarsal axial rotation using the first metatarsal axial radiographs. J Orthop Sci. 2020;25(4):664-70.

16. Lee KB, Kim MS, Park KS, Lee GW. Importance of postoperative sesamoid reduction on the outcomes of proximal chevron osteotomy for moderate to severe hallux valgus deformity. Foot Ankle Surg. 2019;25(4):434-40.

17. Mortier JP, Bernard JL, Maestro M. Axial rotation of the first metatarsal head in a normal population and hallux valgus patients. Orthop Traumatol Surg Res. 2012;98(6):677-83.

18. Saltzman CL, Brandser EA, Anderson CM, Berbaum KS, Brown TD. Coronal plane rotation of the first metatarsal. Foot Ankle Int. 1996;17(3):157-61.

19. Zitouna K, Selmene MA, Khlif MA, Riahi H, Barsaoui M. Effect of sesamoid position on functional outcome of operated hallux valgus. Tunis Med. 2019;97(12):1370-4.

20. Wagner E, Wagner P. Metatarsal pronation in hallux valgus deformity: a review. J Am Acad Orthop Surg Glob Res Rev. 2020; 4(6):e20.00091.

21. Dayton P, Feilmeier M, Hirschi J, Kauwe M, Kauwe JS. Observed changes in radiographic measurements of the first ray after frontal plane rotation of the first metatarsal in a cadaveric foot model. J Foot Ankle Surg. 2014;53(3):274-8.

22. Eustace S, O’Byrne J, Stack J, Stephens MM. Radiographic features that enable assessment of first metatarsal rotation: the role of pronation in hallux valgus. Skeletal Radiol. 1993;22(3):153-6.

23. Gomez Galvan M, Constantino JA, Bernaldez MJ, Quiles M. Hallux pronation in hallux valgus: experimental and radiographic study. J Foot Ankle Surg. 2019;58(5):886-92. 